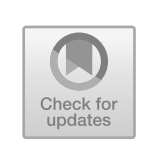

\title{
Task Shifting, Midwifery Empowerment and the Nascence of Clinical Pharmacy
}

\begin{abstract}
This chapter addresses the role that the intervention has played in shaping professional engagement within the multi-disciplinary team. The existence of laboratory results has triggered the emergence of clinical pharmacy roles. The chapter traces the impact of this on prescribing behaviour and on procurement planning and hospital policies. Whilst celebrating the progress made and viability of the model, it describes the structural impact that access to antibiotics and IPC supplies has on the realisation of optimal change.
\end{abstract}

Keywords Antimicrobial stewardship - Maternal sepsis · Task shifting · Clinical pharmacy · Pharmacotherapy · Antibiotic consumption · Antibiotic susceptibility · Procurement planning · Stock-outs

In addition to describing the importance that swabbing and testing have made to progress, the midwife in the previous chapter alludes to a major change in team working and task shifting with midwives and nurses now playing a very central role in these processes. This has been critical to the effectiveness of the MSI. Endorsed by the WHO (2007), task shifting has been implemented in the global health care setting in an effort to combat healthcare worker shortages, increase efficiency and cut costs. In general terms, task shifting is the delegation of a specific task to a lower cadre (WHO 2008). Utilising the available workforce

(C) The Author(s) 2020

L. Ackers et al., Anti-Microbial Resistance in Global Perspective, https://doi.org/10.1007/978-3-030-62662-4_6 
in this manner promotes the more effective and efficient use of human resources. With greater numbers of healthcare staff being able to offer certain aspects of care or perform certain clinical procedures, it follows that there is an increased ability to provide healthcare services, coupled with improvements to healthcare worker skills, greater efficiency within the healthcare system and cost savings (WHO 2007). Task shifting in the West implies an increase in remuneration and substantial investment in continuous professional and career development. The reference to non-medical prescribing ${ }^{1}$ is a case in point. The same cannot be said in LMICs where healthcare professionals are often burdened with additional responsibilities without being compensated accordingly. Nonetheless, task shifting is recommended as a method to reduce healthcare worker shortages in the maternity setting (WHO 2012). Despite a lack of policy framework to support task shifting in Uganda, it has been unofficially practised in the country since 1918 (Baine et al. 2018).

Whilst it is the responsibility of all healthcare professionals to act as antibiotic stewards, we have found that although midwives and nurses are barely mentioned in the NAP, they have, by means of informal task shifting, taken on a significant role in advocating for antimicrobial stewardship. In the Ugandan context, their active engagement and empowerment are absolutely essential to AMS, not least because they are most often the only cadres continually present on the ground. The presence of senior doctors is at best sporadic with rotating and largely unsupervised intern doctors providing most medical input (Ackers et al. 2016; Tweheyo et al. 2019). This evidence adds weight to Brink et al.'s proposal for new nurse-led models of AMS in Africa (2016).

A midwife respondent explains how the new knowledge, combined with team working and support, has facilitated effective task shifting on the ward:

Before [the project] staff did not have the idea about wound swabbing; they didn't understand culture and sensitivity testing. That's where a lot of improvement has come. They now understand the science and take an interest and ask, 'why is this mother not getting better?' They have learnt about resistance. Midwives are now taking the lead in managing patients; they used to wait for doctors to make decisions, but the doctors only appear once in a while, so they waited for them instead of acting. Now

\footnotetext{
${ }^{1}$ See note 31 .
} 
they act. They used to say, 'that one is for the doctor - what has killed this mother?'

The intern doctors on the ward have also observed marked changes in the behaviour of the midwives-and their own practices. One intern doctor commented that, when he arrived:

We found a lot of sepsis on the ward but during that period there was a scarcity of things to use - antiseptics, saline; then the staff started taking off cultures. You have really helped us. Your team has done a lot to reduce sepsis here. They assess the wounds - take off the culture - bring the results as to which microorganisms are resistant, so we cleared all septic mothers.

[Has swabbing practice changed?]

Yes, it has changed - the nurses ${ }^{2}$ do it perfectly now.

[Would you say most wounds are now being swabbed and going to the laboratory?]

In fact, all of them. Once we get a septic mother they will be swabbed. Immediately the first thing is to do a pus swab.

Another intern doctor acknowledges that behaviour change has been evident amongst all cadres:

I really think we did have that knowledge before, but we did not appreciate its significance

[When you say 'we' do you mean the doctors or midwives?]

I think you should consider all of us. We did not do as much as we should have been doing. As long as we dressed the wounds once a day and I prescribed antibiotics the rest of the things would take their course. So, what $\mathrm{K} 4 \mathrm{C}$ has brought to reality is that - actually when this is done, and samples are taken and followed up then things get better more quickly.

[You said before people were aware of culture and sensitivity testing - they had the knowledge but didn't appreciate its significance?]

Exactly. [ $\mathrm{K} 4 \mathrm{C}$ midwives] take the swabs and we get results now in three days. They also get you the pharmacists on the ward so we can target that specific organism.

${ }^{2}$ It is common in Uganda for doctors to use the term 'nurse' generically to include midwives. 
The intern doctor in the quote above notes the role that the midwives play in anchoring pharmacy into the MDTs. One of the most important changes brought about as a direct result of increasing wound management and culture and sensitivity testing has been the way that this has facilitated clinical pharmacy. Since the project commenced, pharmacy has been directly involved in patient management and prescribing decisions as part of a proactive multi-disciplinary team. It is important to note that the pharmacists were fully aware of the benefits of this approach prior to the project and had taken steps to actively encourage it. There was no evidence of a knowledge gap as such. However, until this became a reality on the ward, their ability to utilise their expertise in clinical pharmacy was limited. Chapter 1 reviewed predefined metrics set out by the funding body. Pharmacists on the ground were highly sceptical of the value of antibiotic consumption metrics in this environment stating that:

We cannot measure performance by zeroing on antibiotic [consumption] only.

They proposed instead what they felt was a more relevant indicator, namely the 'Review of Pharmacotherapy' by pharmacists. We are concerned here with the extent to which pharmacists are directly engaged in multi-disciplinary decision-making following the receipt of laboratory test results. Prior to the MSI, it was rare to have pharmacists present on the ward. A K4C midwife explains the importance of the culture and sensitivity testing process to the harnessing of clinical pharmacy engagement:

Pharmacy will tell you there is no point in them coming unless there are cultures.

The data collated from patient notes showed that, once the intervention was in place, pharmacists reviewed the pharmacotherapy in $91.8 \%$ of cases where test results were taken and showed a bacterial growth. ${ }^{3}$ The regular presence of pharmacy on the ward has in turn formed a critical part of the mentoring and knowledge transfer amongst the whole team (and explicitly including the $\mathrm{K} 4 \mathrm{C}$ staff and $\mathrm{UK}$ volunteers). One of the pharmacists

${ }^{3}$ Patient Records (January 2019-January 2020). 
describes the changes in practice and the role that laboratory results have played in this:

Culture and sensitivity testing has been very important especially in post - c-section sepsis. We need to know what we are dealing with. Antibiotic selection is a big issue in AMR. Before they were making a blind selection. They were using only the antibiotics they were used to, the standard arrangement of routine antibiotics. Patients were staying for an extended period of time; the drugs were not working. They never figured it out that they were using the wrong antibiotics. Now it has become standard practice that you take a sample and send it to the laboratory and wait for the results to come. We have now moved to individualised antibiotic selection based on the laboratory results. We know what [the infection] is susceptible to.

He goes on to outline the need for more support through informal mentoring and formal training to enable all cadres to interpret the laboratory reports:

Most of the problems now are how to interpret and apply the [lab.] reports. It is important we all agree on the value of that report and how to interpret it and do things in accordance with the findings.

The project responded to this explicit request for training by organising a small session for staff on the PNG ward. Later, the pharmacy team took the initiative to organise a larger training workshop for representatives of the entire hospital at a morning meeting with intern pharmacists taking the lead.

The MSI has achieved optimal pharmacy engagement (in a $\mathrm{RRH}$ context) on the PNG ward. The impact of laboratory testing has played an important role in empowering pharmacy. This is evident in the new policy, initiated by pharmacy with strong support from the laboratory, of only permitting use of high-end antibiotics when laboratory test results are available:

Clinicians are not allowed to change antibiotics now without cultures.

Discussions have taken place in the hospital's IPC committee to extend this policy to all wards illustrating the wider impact of the MSI on the 
hospital. The laboratory respondent welcomes this achievement which reflects the growing recognition of pharmacy in the hospital:

The policy of only prescribing high end antibiotics to patients who have had culture and sensitivity testing has really worked; these antibiotics are being guarded jealously now. In fact, (the pharmacy team) are very strict on that. I really feel this could work on other wards. It is only working on post-natal ward at present because they have laboratory reports.

The extent to which microbiology results have been understood, valued and applied by midwives on the ward is exemplified in the following excerpt:

After Christmas (2019) we didn't have many septic patients but a week after four of them had the same isolates with the same results. We wondered how come four people worked on separately can come in with the same isolates? They came from different places. How can we get to know the source? We took the swabs off immediately. We did discuss with the laboratory people, but we were concerned so we looked at it ourselves. Is it the hygiene of the patients or the surroundings? Are there other possibilities? We know it is a HAI; Acinetobacter is not an organism you can find outside the hospital. The challenge is it is airborne - we spoke to the pharmacist and the laboratory. Having 4 cases with the same isolate is more of an outbreak. Maybe the laboratory could swab surfaces, or we could fumigate?

Ultimately, the model that has evolved on PNG ward has demonstrated the potential to significantly reduce infection, improve prescribing practice, reduce antibiotic consumption and overcome some of the effects of AMR on patient outcomes. To that extent, significant progress has been made to put systems in place to facilitate evidence-based prescribing. Ultimately, the impact of these systems hinges on access to antimicrobials.

Strategic Objective Three (NAP) highlights the issue of Optimal Access to essential antimicrobials and states very clearly that, 'the major modifiable driver of AMR is the use of antimicrobial agents' (p. 14). Sub-objectives focus on distribution and supply chain mechanisms. 


\section{Procurement and Supply Chain Dynamics in Regional Referral Hospitals}

The following section outlines critical problems in the supply chain system influencing the availability of antibiotics in Ugandan Regional referral hospitals. These include:

1. Budget Constraints: the hospital may only order against a budget prescribed by the Ministry of Finance and held centrally by National Medical Stores (NMS).

2. Limited Options: the hospital can only order antibiotics from a prescribed catalogue which excludes many of the antibiotics indicated as necessary from laboratory results and present on the Essential Medicines and Health Supplies List for Uganda (EMHSLU).

3. Failure to deliver on orders: major and unpredictable discrepancies exist in what is ordered and what is delivered ('Order Fill Rates').

4. As a result of the above, most IPC supplies and antibiotics run out half-way through the bi-monthly supply cycle (Stock-Outs).

In Uganda, the funds for procurement of drugs and supplies in the public sector are highly centralised and inadequate. NMS procures and distributes supplies to health facilities based on a centrally allocated Annual Supplies Budget. Each hospital is required to produce an Annual Procurement Plan. Once agreed, this Plan is fixed and cannot be varied over the year reducing the opportunity for flexibility and responsiveness to the hospital laboratory results and any changes indicated by a future antibiogram. This budget is held by NMS. With the exception of private wards, it is not possible for a RRH to source supplies from elsewhere. NMS deliveries take place bi-monthly.

During the annual procurement process, the hospital may only order those items authorised by the EMHSLU. However, not all essential drugs feature in the NMS catalogue. A hospital pharmacist describes the situation as follows:

As much as we may desire a certain antibiotic, we can't plan for it if it is not present in the catalogue. A case in point is Amikacin and Moxifloxacin.

Out of the nine antibiotics tested against Acinetobacter samples in the laboratory, only two, doxycycline and amikacin, showed greater levels 
of susceptibility than resistance. Given the much higher success rate of amikacin, it is paramount that the antibiotic can be obtained for cases of severe Acinetobacter infections where other avenues have failed.

When the project started, the procurement plan for the financial year $2018 / 2019$ was already in place. At that time, the plan did not take account of antibiotic susceptibility as reported by the laboratory, resulting in a lack of high-end antibiotics required to treat resistant bacterial strains. The evidence generated through the MSI, supported by the engagement of the $\mathrm{K} 4 \mathrm{C}$ pharmacist, contributed to the procurement planning process for 2020/2021. Table 6.1 presents an extract from the hospital's procurement plan to indicate key antibiotics used on the post-natal and gynae ward. It evidences significant changes in antibiotic ordering and consumption arising directly from the intervention, where antibiotics in red denote project-related increases and those in blue denote decreases. For now, ${ }^{4}$ we draw the reader's attention to column 5 (average past consumption). This shows the predominance of a small number of antibiotics including amoxicillin, metronidazole, ciprofloxacin, ceftriaxone and chloramphenicol.

\section{Antibiotic Distribution at Ward Level in FPRRH}

When supplies arrive at FPRRH from NMS, they are taken to the main stores. At this point, supply data is recorded electronically in the online ordering system $(\mathrm{Rx})$. When we first visited pharmacy stores at the start of the project, we found the $\mathrm{Rx}$ system unused. The $\mathrm{Rx}$ initiative is a national programme designed to improve supply chain management and provide an online database linking the hospitals to NMS. When functional, it would provide a systematic method of stock utilisation and recording providing high-quality data to the hospital. It was clear that the pharmacy team had the expertise to use the software but there was no printer ink available. Two donated printers sat unused as they had no ink and obtaining cartridges for these was very complex. This illustrates the problems of managing donated devices in LMICs especially when they are not integrated in any way to local supply chains. The hospital had ink in stock but for very different printers and could not provide the printer ink to the stores. In this instance, the MSI provided a printer and printer ink

\footnotetext{
${ }^{4}$ We return to discuss the impact of the MSI on Procurement Planning in due course.
} 
Table 6.1 Extract from the 2020/2021 procurement plan (with bi-monthly figures) focusing on key antibiotics used on PNG wards ${ }^{\mathrm{a}}$ at FPRRH

\begin{tabular}{|c|c|c|c|c|c|c|}
\hline & Unit & Price & $\begin{array}{c}2019 / 2020 \\
\text { plan }\end{array}$ & $\begin{array}{l}\text { Past Av. } \\
\text { consumpt. }\end{array}$ & $\begin{array}{c}2020 / 2021 \\
\text { plan }\end{array}$ & $\begin{array}{c}\text { Bi-monthly } \\
\text { cost }\end{array}$ \\
\hline $\begin{array}{l}\text { Amoxicillin } 250 \mathrm{~g} \\
\text { capsules }\end{array}$ & 1000 & 5,4100 & 101 & 134 & 90 & $4,869,000$ \\
\hline $\begin{array}{l}\text { Ampicillin/cloxacillin } \\
\text { capsules }\end{array}$ & 100 & 14,400 & 30 & 42 & 50 & 720,000 \\
\hline $\begin{array}{l}\text { Metronidazole } \\
\text { tablets }\end{array}$ & 1000 & 17,100 & 50 & 70 & 70 & $1,197,000$ \\
\hline $\begin{array}{l}\text { Chloramphenicol } \\
250 \mathrm{mg} \text { tabs }\end{array}$ & 1000 & 10,0200 & 0 & 0 & 5 & 501,000 \\
\hline $\begin{array}{l}\text { Ciprofloxacin } 50 \mathrm{mg} \\
\text { tablet }\end{array}$ & 100 & 10,500 & 70 & 118 & 70 & 735,000 \\
\hline Ceftriaxone $1 \mathrm{~g}$ vials & 1 & 1700 & 6000 & 6000 & 5000 & $8,500,000$ \\
\hline $\begin{array}{l}\text { Ampicillin/cloxacillin } \\
250 \mathrm{mg} \text { inj }\end{array}$ & 50 & 33,000 & 0 & 2 & 1 & 33,000 \\
\hline $\begin{array}{l}\text { Meropenem 500mg } \\
\text { inj }\end{array}$ & 100 & 71,700 & 2 & 5 & 2 & 143,400 \\
\hline $\begin{array}{l}\text { Gentamycin 80mg } \\
\text { inj }\end{array}$ & 1 & 16,000 & 0 & 92 & 150 & $2,400,000$ \\
\hline $\begin{array}{l}\text { Ciprofloxacin IV } \\
\text { 200mg }\end{array}$ & 100 & 32,800 & 40 & 0 & 0 & 0 \\
\hline $\begin{array}{l}\text { Metronidazole } \\
500 \mathrm{mg} \text { infus }\end{array}$ & 50 & 33,000 & 100 & 217 & 150 & 195,000 \\
\hline Cefotaxime inj & 1 & 800 & 7000 & 5833 & 7000 & $5,600,000$ \\
\hline $\begin{array}{l}\text { Penicillin } \\
\text { Benzathene } 2.4 \mathrm{MU} \\
\text { amp }\end{array}$ & 1 & 13,400 & 1 & 767 & 0 & 0 \\
\hline \multirow{2}{*}{$\begin{array}{l}\text { Chloramphenicol lg } \\
\text { inj. }\end{array}$} & 10 & 11,400 & 5 & 4 & 5 & 57,000 \\
\hline & 50 & 80,200 & 0 & 0 & 5 & 401,000 \\
\hline
\end{tabular}

an this table, the column 'unit' shows the number of doses per unit as sold. 'Price' is the price per unit in Ugandan Shillings (1 USD $=3780$ UGX, May 2020). The column '2019/2020 plan' shows the number of units that were ordered for delivery every other month in 2019 , with 'past av consumption' detailing the average bi-monthly consumption of the past year for each antibiotic. The column '2020/2021' details the set number of units ordered every other month for this year, with the corresponding bi-monthly cost reported in the final column

Source Hospital procurement plan, 2020-2021

for the duration of the project to enable the $\mathrm{Rx}$ system to become part of the organisational culture in pharmacy stores. Since then, the hospital has employed a dedicated stock manager to work with this system freeing up the senior pharmacist to engage in clinical pharmacy roles. We have 
discussed what may seem a mundane supplies issue in some depth here as this is precisely the kind of problem that lies behind major systems failures in Uganda, the diseconomies of 'donations' and the ease with which organisations become dependent on donor support. It also illustrates one of the underlying causes of data quality problems.

The process of distributing supplies within the hospital is, unfortunately, not presently covered by the $\mathrm{Rx}$ electronic system. Instead, the in-patient pharmacy (located a short distance from the stores) orders from the stores. Individual wards then visit the in-patient pharmacy to requisition supplies, and this is recorded manually on forms and in a records ledger book (the HMIS Dispensing Log).

Figure 6.1 presents data obtained from the in-patient pharmacy on the distribution of oral antibiotics between the main hospital wards. We recorded this data for the months of January and February 2020 to gain a picture of the distribution of injectable and oral antibiotics over the 2-month supply cycle and put PNG ward consumption patterns in a whole-hospital context. As can be seen, the level of antibiotic consumption on the PNG wards as a proportion of overall consumption is high and indicates the importance of this to overall antimicrobial stewardship:

Tables 6.2 and 6.3 identify antibiotic consumption on the PNG wards in more detail over a 4 -month window. Table 6.2 provides data for Intravenous Antibiotics and evidences the predominance of metronidazole and ceftriaxone. Table 6.3 provides data for Oral Antibiotics and shows the

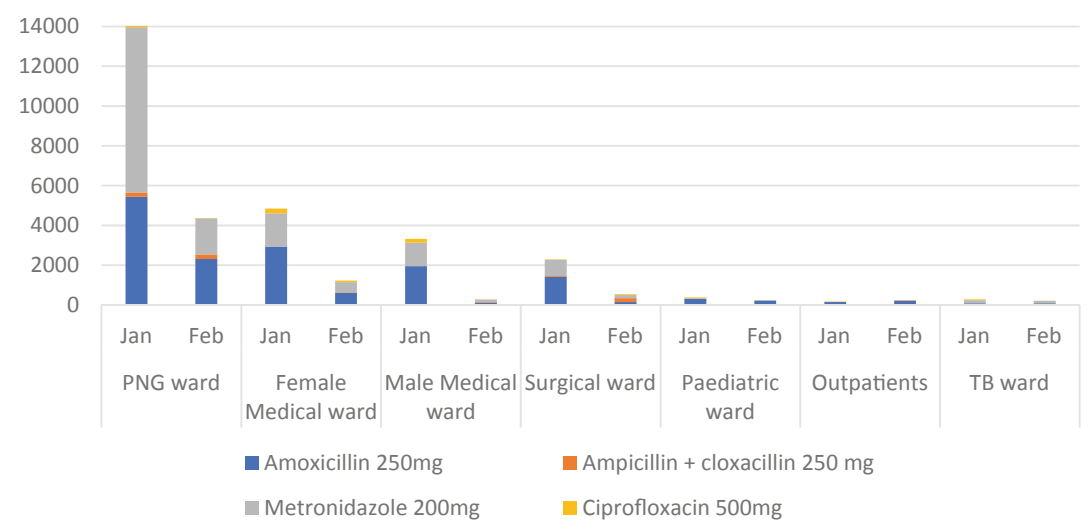

Fig. 6.1 Supply of oral antibiotics to all wards in January and February 2020 
6 TASK SHIFTING, MIDWIFERY EMPOWERMENT ...

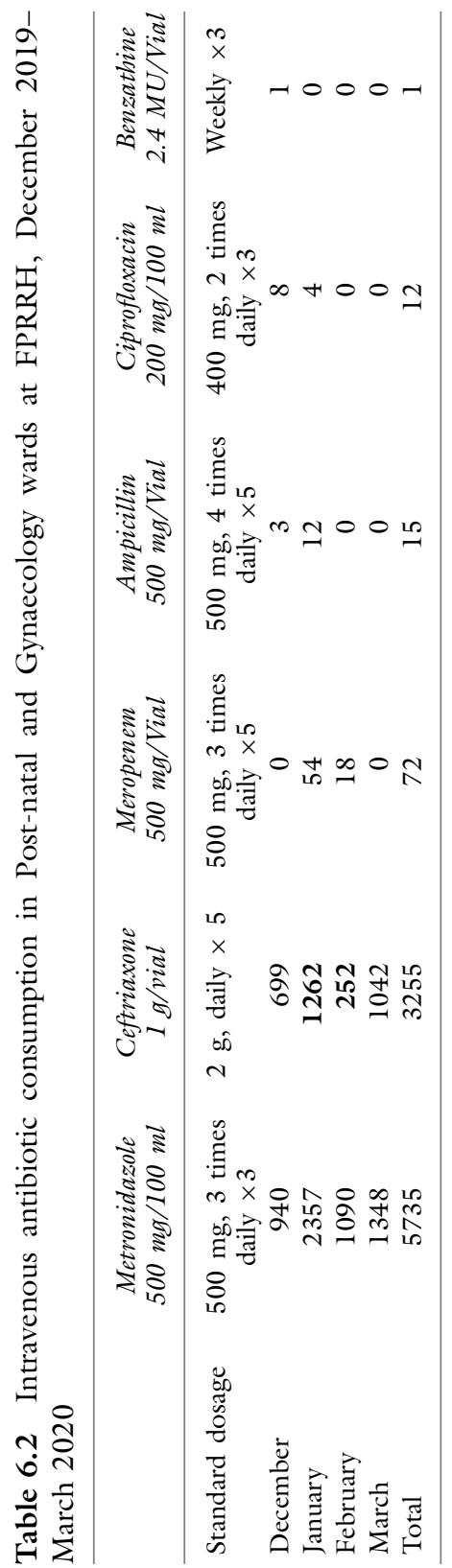


Table 6.3 Oral antibiotic consumption in post-natal and Gynaecology wards at FPRRH, December 2019-March 2020

\begin{tabular}{|c|c|c|c|c|c|c|}
\hline & $\begin{array}{l}\text { Metronidazole } \\
200 \mathrm{mg} \text { tabs }\end{array}$ & $\begin{array}{l}\text { Amoxicillin } \\
200 \mathrm{mg} \\
\text { caps }\end{array}$ & $\begin{array}{l}\text { Ampicillin } \\
\text { \& } \\
\text { Cloxacillin } \\
500 \mathrm{mg} \\
\text { caps }\end{array}$ & $\begin{array}{l}\text { Ciprofloxacin } \\
500 \mathrm{mg} \text { tabs }\end{array}$ & $\begin{array}{c}\text { Doxycycline } \\
100 \mathrm{mg} \\
\text { caps }\end{array}$ & $\begin{array}{c}\text { Cefuroxime } \\
500 \mathrm{mg} \\
\text { tabs }\end{array}$ \\
\hline $\begin{array}{l}\text { Standard } \\
\text { dosage }\end{array}$ & $\begin{array}{l}400 \mathrm{mg}, 3 \\
\text { times daily } \times \\
5\end{array}$ & $\begin{array}{l}400 \mathrm{mg}, \\
3 \text { times } \\
\text { daily } \times 5\end{array}$ & $\begin{array}{c}500 \mathrm{mg} \text {, } \\
2 \text { times } \\
\text { daily } \times 5\end{array}$ & & $\begin{array}{l}100 \mathrm{mg}, 2 \\
\text { times daily } \\
\quad \times 7\end{array}$ & $\begin{array}{c}1000 \mathrm{mg}, \\
3 \text { times } \\
\text { daily } \times 5\end{array}$ \\
\hline December & 7477 & 2400 & 732 & 410 & 144 & 0 \\
\hline January & 8298 & 5436 & 220 & 102 & 182 & 10 \\
\hline February & 1812 & 2308 & 235 & 20 & 153 & 0 \\
\hline March & 7474 & 4860 & 0 & 650 & 88 & 0 \\
\hline Total & 25061 & 15004 & 1187 & 1182 & 567 & 10 \\
\hline
\end{tabular}

dominance of metronidazole and amoxicillin. Both sets of data show large fluctuations in consumption over the 4-month period with particularly depressed consumption in February:

The fluctuations identified above suggest serious supply issues determining access to antibiotics. With current systems in place, the major challenge facing the MSI model is access to the right antimicrobials at the right time. The laboratory scientist is clear about this:

Antibiotic stock-outs remain a serious constraint; in many cases patients can only be given the right antibiotics if they pay and many of them can't pay. We have to be very clear, antibiotic stock-outs are a key factor fueling AMR. If we look at the scenario where we have investigations done and antibiotics are available, and the outcomes are good, but we have done the investigations and the antibiotics are out of stock we won't have a good outcome.

There is a bigger concern here too; if supplies are not available and the ward staff are unable to respond effectively to laboratory results, this can be predicted to have a major impact on staff motivation and the behaviour change gains achieved. Problems of access critically restrict pharmacy's ability to engage in rational prescribing (prescribing the drug most likely to work according to laboratory results). This in turn leads to over-consumption of poorly performing (and often cheaper) antibiotics 
and poor patient outcomes. The pharmacy team involved in procurement planning pointed out the severe budgetary constraints they faced when attempting to order new antibiotics in response to laboratory results. In practice, this meant making difficult 'trade-offs', especially when the new antibiotics are so much more expensive than those they were able to reduce. The reduction in supply of amoxicillin, for example, is explained as follows:

We realised that the majority of patients using amoxicillin were mothers discharged after giving birth. They are usually given amoxicillin as prophylaxis to prevent infection. Some were being given for a longer duration than necessary. As pharmacy staff, we intervened so that the duration of treatment would be reflective of the nature of risk. This led to a reduction in use. We had to increase certain antibiotics or include new antibiotics as well. Due to budget constraints, it was agreed during the planning stage that we cut on the quantity of Amoxicillin to free up some budget to cater for other needed antibiotics.

The laboratory results indicated very high levels of resistance to both amoxicillin and ampicillin, both of which are derived from penicillin. Based on these assumptions, ampicillin and amoxicillin will have minimal effects on the three primary bacterial causes of infection. Significant changes in planned use of meropenem can also be directly attributed to the MSI, where imipenem was shown to be $100 \%$ effective against E. coli. The pharmacist explains that consumption of this drug over the past year has been reliant entirely on donated supplies (it was not ordered in 2019):

[The increased order] can be supported by evidence generated by the laboratory. Due to the increased culture and sensitivity reporting, we noticed that there was improved sensitivity to meropenem. This ensured that we were able to convince members involved in planning to include it on the $2020 / 2021$ plan. We were able to get some donations last year and that's why it shows that we consumed it. What's more, we wrote to NMS to allow us to procure it, even though it's not in the current plan.

This action, of communicating directly with NMS on procurement, represents one example of where the pharmacy team have attempted to advocate as a result of the MSI. The impact of this procurement may be to the benefit of other hospitals if NMS are influenced to place it on their catalogue in future. The decision to increase orders of meropenem 
required the team to make stark choices which led to the reduction in orders of cefotaxime:

Just like Meropenem, this particular consignment of Cefotaxime (used in 2019) was a donation. It wasn't in the procurement plan. While working on the 2020/2021 plan, we had to prioritise between Cefotaxime and Meropenem. We had to go with Meropenem. We did factor in the cost and resistance profile per the lab reports.

Cefotaxime has shown high levels of resistance in the laboratory tests. The marked rise in procurement of ciprofloxacin is also directly attributable to the MSI although the pharmacy team were concerned about the volume needed:

What we require is actually a lot more. Again, [the increase] can be explained by the results of culture and sensitivity. There seems to be less resistance to ciprofloxacin.

The pharmacist sums up the impact that the project has had on procurement planning and the constraints the team had to work with:

We had to reduce the quantity of Ceftriaxone by a significant margin. This was supported by laboratory data which showed a lot of resistance to ceftriaxone. Some of the monies freed up were used to plan for chloramphenicol and meropenem, drugs which are showing less resistance as per lab reports. There is no significant increase in the incoming budget for drugs and medical sundries. It's therefore painstaking to reallocate priorities in terms of drugs while maintaining the same budget. Our [MSI] efforts to encourage and support Culture and Sensitivity testing and sharing this with the procurement planning team lead us to include some much-needed antibiotics (Meropenem and Chloramphenicol) in next year's plan and reduce the procurement of antibiotics with a lot of resistance (Ceftriaxone).

Table 5.11 also provides an indication of the cost implications of the changes in antibiotic procurement because of the MSI. Most of the increases in procurement involve more expensive antibiotics. The procurement plan reflects the negotiations the pharmacy team have engaged into balance the need for rational prescribing against the cost implications of 
Table 6.4 Order fill rate at FPRRH $(2019 / 2020)$

\begin{tabular}{lccc}
\hline Financial Year Cycle & Total Items Ordered & Total Items Delivered & $\begin{array}{c}\text { Fill rate } \\
(\%)\end{array}$ \\
\hline $\begin{array}{l}\text { CYCLE 1 (July-August } \\
2019\end{array}$ & 307 & 236 & 77 \\
$\begin{array}{l}\text { CYCLE 2 } \\
\text { (September-October }\end{array}$ & 306 & 232 & 76 \\
$\begin{array}{l}\text { 2019) } \\
\text { CYCLE 3 } \\
\text { (November-December }\end{array}$ & 309 & & \\
2019) & & 226 & 73 \\
\hline
\end{tabular}

Source $\mathrm{Rx}$ on-line medicines management system (NMS do not provide data on fill rates for specific medications)

buying more expensive antibiotics. Unfortunately, the constraints of the NMS budget-line are not the end of the story.

\section{Discrepancies Between Order AND SUPPly (ORDER Fill Rates)}

In practice, not all that is ordered by the hospital from NMS is supplied. The 'Order Fill Rate' gauges the delivery performance of total number of items ordered against the total number of items delivered. As clearly seen in Table 6.4, NMS supplies about 75\% of orders. More specific discrepancies may also arise. Unusually, NMS failed to deliver ceftriaxone in September 2019, for example.

\section{Key Challenges to Sustained Behaviour Change: The Impact of Stock-Outs on AMS}

Stock-outs (the exhaustion of supplies) are a feature of Ugandan public health facilities at all levels and a major factor contributing to sepsis deaths in maternal and new-born health (Bua et al. 2015). Inevitably, bi-monthly deliveries tend to be exhausted quite rapidly and often by the end of the first month:

For example, when we get 6000 vials of Ceftriaxone, we consume all of it in maybe 4 weeks, then we stay without for another 3-4 weeks. And the 
following cycle, we get the same quantity. Therefore [consumption data] are merely an average of what is not enough.

Stock-outs are caused by a combination of misuse and overall shortfalls. The pharmacists noted that the project had improved antibiotic use on the wards:

When these antibiotics are received [from NMS] they tend to run out quickly. Again, this is attributed to the small budget and probably misuse/irrational prescribing. However [MSI's] endeavour to link the ward, pharmacy and the Lab has to a great extent solved the issue of irrational antibiotic prescribing.

The issue of stock-outs remains a serious block to progress. An intern doctor describes the shortages affecting his work that are likely to result in Surgical Site Infections:

We always have challenges with supplies from National Medical Stores (NMS). We have them, then for 2 months we don't have any. They deliver every quarter but in the last 6 weeks we have none - there is no oxytocin now. We have no iodine in theatre, so we are just using saline and during that time we need some help until NMS provide for us. We order for 3 months but things get used before 6 weeks. These are the challenges that make us get sepsis on the ward.

To illustrate the breadth of this shortfall, on February 18th, 2020, the PNG ward contacted K4C to request support in the purchase of gauze. Without this, they would not be able to continue with the wound dressing established on the ward. This would have resulted in increased infection and sepsis (and antibiotic consumption). We were aware during the project visit in January 2020 that the hospital had also run out of disinfectants, iodine and spinal needles (amongst many other things). In such circumstances, the only option is for staff to ask patients to pay for the necessary items, and if they are unable to pay, then operations will not happen, and major delays occur in treatment. On February 19th, 2020, we established that 13 key items for use on the PNG ward were out of stock and had been for over a month:

1. Ceftriaxone injection

2. Intravenous metronidazole 


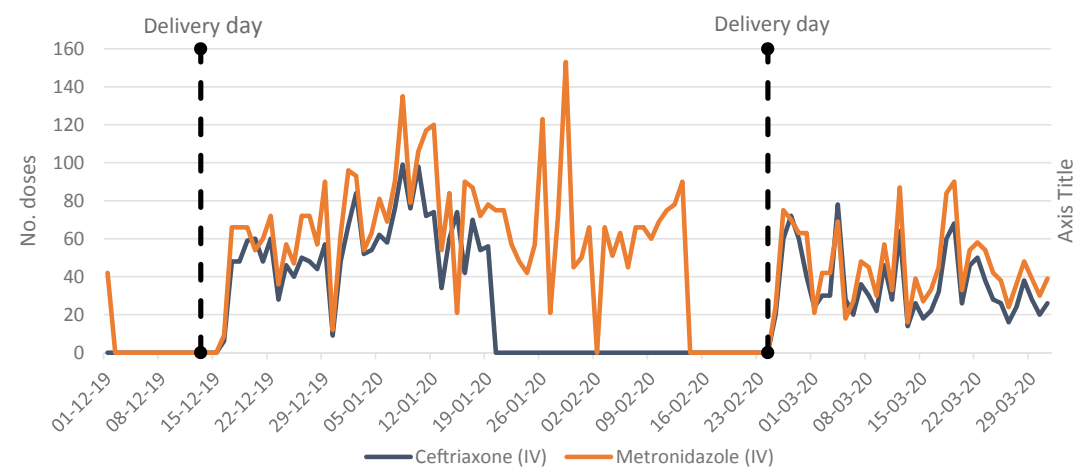

Fig. 6.2 Dispensing of IV Ceftriaxone and Metronidazole from in-patient pharmacy to PNG wards at FPRRH (1 December 2019-29 March 2020)

3. Intravenous normal saline $0.9 \%$

4. Intravenous ringers lactate

5. Intravenous ciprofloxacin

6. Meropenem $1 \mathrm{~g}$ injection

7. Gentamicin $80 \mathrm{mg}$ injection

8. JIK (sodium hypochlorite) solution

9. Alcohol hand gel

10. Chlorhexidine gluconate $4 \%$

11. Cotton wool $1 \mathrm{~kg}$ (hospital quality)

12. Gauze (hospital quality)

13. Povidone Iodine.

The next supplies were expected on February 25th, 2020.

We have listed the items to illustrate the profound impact of such stock-outs on ward cleaning; hand hygiene ${ }^{5}$; the ability to clean and dress wounds and prescribe necessary antibiotics. Figures 6.1 and 6.2 present data collected manually from in-patient pharmacy on key antibiotics used in the PNG wards. NMS deliveries were made on December 13th, 2019, and February 24th, 2020. The dispensing patterns show stark evidence of stock-outs.

${ }^{5} \mathrm{~K} 4 \mathrm{C}$ makes up this shortfall. 
The greater consumption of metronidazole can be attributed to the high dosing regimen (three times daily) plus its empirical indication as a broad-spectrum therapy for prophylaxis against anaerobes. IV Ceftriaxone (dosed once daily) is also being used empirically and for prophylaxis, especially in surgical cases. IV Ceftriaxone was not available at all from February 19th to March 1st. Dispensing of IV metronidazole showed evidence of stock-outs but for shorter periods than ceftriaxone. In the period between February 15th and 23rd, neither IV metronidazole nor IV ceftriaxone were available to the PNG wards. Similar falls can be seen at Christmas and New Year. These dips we would anticipate being due to lack of doctors on the wards to prescribe (so a human resource management issue rather than a stock-out issue) (Fig. 6.3).

Dispensing patterns for oral metronidazole show higher utilisation and longer periods of stock-outs than oral amoxicillin, with an extended stockout from January 26th to February 23rd coinciding exactly with the stock-out of IV metronidazole.

The interview with the hospital administrator raised this issue. $\mathrm{He}$ explains the lack of autonomy the hospital has in relation to procurement:

[Interviewer] The biggest blockage seems to be stock outs of antibiotics, jik and disinfectant. It seems when they deliver after 1 month it is all gone so you have a month with none so even if we do the swabbing and testing if the right antibiotics are not in stock, we can't do anything. It seems you

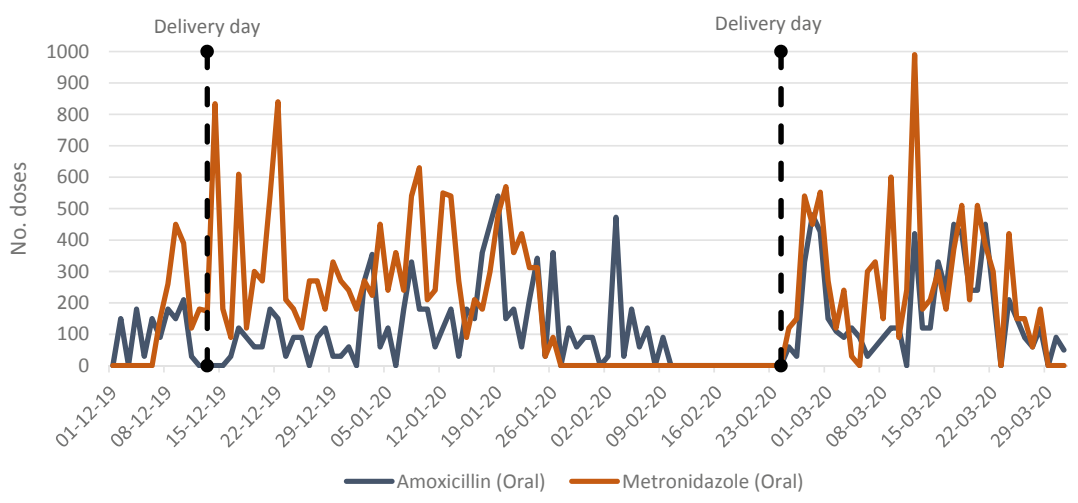

Fig. 6.3 Dispensing of oral Amoxicillin and Metronidazole from in-patient pharmacy to PNG wards at FPRRH (1 December 2019-29 March 2020) 
are saving a lot of money, but we are not allowed to use this on drugs and consumables. If that doesn't improve, I don't think we can make any more improvement. Do you have any ideas?

Well NMS should be providing. The funds for buying medicine don't come from our account - it comes from NMS. We are tied to NMS.

Concerns about stock-outs were also raised in the IPC Committee. It was interesting to observe that this issue is so endemic and structural that it was almost not listed in the meeting minutes and would not have been if one of the $\mathrm{K} 4 \mathrm{C}$ team did not raise it. Concern was also expressed by a doctor at the IPC meeting about overuse of antibiotics in some departments contributing to shortages: 'Can we stop some departments using antibiotics for everything - coughs ... malaria...' A senior nurse participant echoed this concern referring to the practice of prolonged antibiotic use: 'clinicians prescribe antibiotics for 10 days then the clinician just says 'continue'. This is one of the practices that we have noticed to have changed on PNG since culture and sensitivity testing became part of the ward's normal practice.

The $\mathrm{K} 4 \mathrm{C}$ pharmacist responded to these concerns by expressing the need for the full institution of a Medicines Therapeutic Committee (MTC) which would have authority to implement clearer policies on antibiotic use.

\section{Interpreting In-Patient Pharmacy Data-A Note}

Consumption data cannot be interpreted in any direct way as an indication of prescribing knowledge or stewardship competency. Collecting and analysing the data on antibiotic 'consumption' patterns at FPRRH have emphasised the dangers of empiricist approaches to data analysis and presentation and the importance of interrogating data rigorously. In most cases, data presents a myriad of questions and very few obvious and immediate answers. This is especially the case when attempting to collate data from public health facilities in Uganda and many other LMICs. Collecting data has been a continuous process of trial and error merging with the underlying ethnographic journey. As described, in the case of in-patient pharmacy, the data is not yet managed electronically via the Rx system. Rather, individual wards come to a window in the in-patient pharmacy with paper forms requesting supplies for that day. The pharmacy then maintains a handwritten record of dispensing in a records book, and 
this data is compiled into forms for the Ministry of Health. In the first instance, we believed that all wards behaved in this manner, but the initial data suggested otherwise. The TB ward, for example, appeared to collect very few drugs. We found that most of the drugs used on the TB ward are in fact provided by a donor ${ }^{6}$ and follow a different track. These drugs pass through the main stores and are directly requisitioned by the TB ward. As such, they do not pass through in-patient pharmacy and neither are they recorded as received from NMS on the $\mathrm{Rx}$ system, leaving a gap in overall supply and consumption data.

Other apparent 'discrepancies' in the data, including very sporadic and low use of antibiotics in the neonatal intensive care unit (NICU) and the paediatric ward, uncovered variances in practice that are undocumented and apparently do not comply with the published protocol. This was explained by one respondent as follows:

The paediatric ward gets injectable drugs (directly) from main stores, including antibiotics, but oral antibiotics and other oral drugs from in-patient pharmacy.

Our observational work on the wards supplemented by qualitative interviews and many emails led us to question the relationship between this 'consumption' data and overall consumption patterns. We know, for example, that since laboratory results have been available, many women are getting higher-end antibiotics and that this is contributing to shortened stays and improved patient outcomes. However, the supplies of these drugs were not visible in the data. It seems that the pharmacists have played a critical role in supporting access to these antibiotics through a combination of 'borrowing' from other hospital supplies. The following excerpt explains this process:

There is a TB focal person who handles all TB related logistics, including the ordering of $\mathrm{TB}$ drugs. These drugs are stored in main stores. $\mathrm{Rx}$ only focuses on drugs from NMS. In most cases, we don't have the changed antibiotics in stock or in our procurement plan or we have limited quantities. Take Amikacin for example. We usually borrow from the TB

${ }^{6}$ The Ministry of Health's National Medicines Policy reports that donor support for medicine financing is more than three times the amount the government invests and concludes that, 'heavy dependence on donor funds puts Uganda in a vulnerable position (2015: 7)'. 
program and give to the patients. The same applies to Moxifloxacin. These medicines are not available in the inpatient pharmacy. In fact, one time you had to use K4C money to purchase Amikacin. Because at times we have septic patients who are only responsive to these drugs, we 'beg'/borrow from the TB drugs. Apparently, this has caused audit queries.

This 'borrowing' behaviour clearly saves women's lives; it also compromises the pharmacists under pressure to assist, but potentially contravening donor conditionalities. We can anticipate similar situations in relation to Anti-Retroviral Therapies (for HIV patients) and, in the case of FPRRH given its proximity to Congo, some (necessary) stockpiling in the event of Ebola spread.

\section{Pharmacy Support in Accessing AND Distributing Antimicrobials}

It is important to recognise the considerable efforts the pharmacy team have made to optimise the distribution of and access to supplies within the hospital. Improved relationships with pharmacy have significantly improved the ability of staff in the PNG ward to access consumables and medicines. The hospital pharmacist explains his role and how he has tried to improve timely access:

I was very happy to be part of the project; most of the things I was trying to do beforehand but I was focused in stores on the supply chain. This is a key role for hospital pharmacists in Uganda. Making sure the necessary consumables are available; that people are using standardised protocols for theatre, but they often don't have consumables. I have a level of authority to ensure consumables are available; to develop good pharmacy distribution practice. The hospital has supplies for different programmes (ART, TB and emerging diseases such as Ebola). I can help to move items around to ensure user access and good distribution. I can ensure different wards have access to medicine so they can do their job. I am involved in proper procurement planning for the entire financial year; how do we move drugs to the user points? I think the commodities for sepsis need to be ring fenced somehow. Some come under different programs; we use one antibiotic that comes here under TB, so we redirected it, and this has been very beneficial. 
Table 6.5 Patients receiving the prescribed antibiotic dose

\begin{tabular}{lcc}
\hline & Gynaecology $(n=22)$ & Post-natal $(n=20)$ \\
\hline No. of patients on antibiotics & 10 & 18 \\
No. of patients documented to have & 4 & 0 \\
received all prescribed doses in last & & \\
24 hours & & \\
\hline
\end{tabular}

Source Results of G-PPS, May 2019 as reported to FPRRH IPC Committee

The midwives have appreciated the lengths pharmacy have gone to try to access antibiotics and consumables:

The relationships with pharmacy are working a lot better; they get us wound dressing materials and [pharmacist] runs to the ward when he is needed.

There was a case who is only sensitive to amikacin. Getting one dose of amikacin is very expensive and our population here cannot afford. It isn't available in the hospital; they can't afford to provide it sustainably as it is very expensive but now [pharmacist] tried hard and we have been able to provide, and she is greatly improved.

When drugs are prescribed but not in stock, patients are asked to buy them privately. ${ }^{7}$ Current recording systems cannot capture the consumption of privately purchased drugs. By way of illustration, the GPPS 'snapshot' found that, on the date of the survey, very few of the patients were receiving the prescribed dose (Table 6.5):

These examples underline the need to exercise caution when interpreting data on antibiotic consumption. We have noted how the presence of laboratory results has facilitated clinical pharmacy on the PNG ward. The presence of one clinical pharmacist has made a major difference. Extending this to other wards may, however, require more pharmacists to be employed.

${ }^{7}$ The regulation of 'over-the-counter availability and self-medication with antimicrobial medicines' is a key stewardship target of the National Action Plan. We were very aware of this huge challenge in Uganda and are undertaking some work to assess the quality of some of these antibiotics. We made the decision not to address this issue in the MSI. Documentation on the ward was also so weak we decided not to attempt to capture the role that private purchases play in antibiotic consumption on the ward. This is an area we would be keen to follow up in future. 


\section{Pharmacy Leadership in a Ugandan Regional Referral Hospital}

Chapter 1 noted the emphasis on pharmacy as a profession in the Call for Applications and, in the 'Scoping Requirements', a preference for pharmacy leadership of AMS. In order to understand the current and potential role that pharmacy, as a profession, can play in the management of AMR, it is important to understand the constraints on pharmacy in context. The role of a hospital pharmacist in a Regional Referral Hospital combines supply chain management with clinical engagement and includes (in theory):

- Monitoring the supply of all medicines used in the hospital, purchasing, compounding, dispensing and quality-testing medication stock.

- Working with the healthcare team to ensure the selection of the best medication at the correct dose for an appropriate duration.

- Monitoring and preventing or minimising side effects and drug interactions.

- Providing medication counselling to patients.

- Dispensing medications for patients in wards, the emergency department and those attending outpatient clinics.

- Offering specialist drug therapy advice to doctors, nurses and other healthcare professionals within the hospital.

When the MSI commenced, there was only one senior pharmacist in the hospital. The Head of Pharmacy in a Regional Referral Hospital also coordinates pharmacy-related activities across the wider region. Fort Portal Regional Referral Hospital is the referral hospital for the Rwenzori region comprising 9 health districts and an approximate population of 3 million people (2014 National Census). ${ }^{8}$

The hospital also employs a pharmacist, who reports to the senior pharmacist. Unfortunately, when the project commenced, the pharmacist had been transferred to the National Referral Hospital. In addition, the hospital hosts intern pharmacists allocated by the National Internship Committee under the Ministry of Health. Internships last 12 months of

\footnotetext{
${ }^{8}$ https://www.ubos.org/2014-census/. The National Medicines Policy reports that only $8 \%$ of pharmacy posts in public service are filled (MOH 2015: 10).
} 
which 6 months must be spent at a hospital with rotations to different sectors including regulatory bodies, community pharmacies and pharmaceutical industries. The hospital usually has four intern pharmacists who rotate through the major wards, pharmacies and stores under the supervision of the pharmacist/senior pharmacist. The hospital also employs two Pharmacy Technicians and two Pharmacy Assistants who are qualified to diploma and certificate level, respectively, and are mainly involved in dispensing. A second pharmacist joined the pharmacy team during the project and was required in the first year of his appointment to develop systems to improve supply chain management. According to the Ministry of Health's 9 plan, there is a vacant position at FPRRH for a principal pharmacist. In this context, there is limited pharmacy capacity to engage more actively in clinical pharmacy across the whole hospital.

This chapter has outlined the opportunities that more active engagement with the laboratory has brought about particularly in stimulating the practice of clinical pharmacy on the wards. It illustrates the gains that can be made from the more effective deployment of just one pharmacist. It has also identified key structural weaknesses in the health system that restrict the opportunity for optimal impact and scale-up. Chapter 7 explores the change process in more detail and the contribution that multi-disciplinary team working has made to knowledge mobilisation and change processes on the ward.

\section{REFERENCES}

Ackers, H. L., Ioannou, E., \& Ackers-Johnson, J. (2016). The impact of delays on maternal and neonatal outcomes in Ugandan public health facilities: The role of absenteeism. Health Policy and Planning, 31, 1152-1161.

Baine, S. O., Kasangaki, A., \& Baine, E. M. M. (2018). Task shifting in health service delivery from a decision and policy makers' perspective: A case of Uganda. Human Resources for Health, 16, 20. https://doi.org/10.1186/s12 960-018-0282-z.

Brink, A. J., van de Bergh, D., Mendelson, M., \& Richards, G. A. (2016). Passing the baton to pharmacists and nurses: New models of antibiotic

\footnotetext{
${ }^{9}$ The MOH allocates staff to Regional Referral Hospitals centrally; hospitals have little autonomy in recruitment.
} 
stewardship for South Africa, South African Medical Journal, 106(10), 947-948.

Bua, J., Mukanga D., Lwanga M., \& Nabiwemba, E. (2015). Risk factors and practices contributing to newborn sepsis in a rural district of Eastern Uganda: A cross sectional study. BMC Res Notes, 8, 339. Published online 2015, August 9. https://doi.org/10.1186/s13104-015-1308-4.

Ministry of Health, Uganda. (2015). Uganda National Medicines Policy.

Tweheyo, R., Reed, C., Campbell, S., et al. (2019). 'I have no love for such people, because they leave us to suffer': A qualitative study of health workers' responses and institutional adaptations to absenteeism in rural Uganda. BMJ Global Health, 4, e001376. https://doi.org/10.1136/bmjgh-2018-001376.

World Health Organisation. (2007). Task shifting to tackle health worker shortages. Retrieved from: http://www.who.int/healthsystems/task_shifting_book let.pdf. Accessed 4 March 2017.

World Health Organisation. (2008). Task shifting. Global recommendations and guidelines. Geneva: World Health Organisation.

World Health Organisation. (2012). Optimizing health worker roles to improve access to key maternal and new-born health interventions through task shifting. Retrieved from: http://apps.who.int/iris/bitstream/handle/10665/77764/ 9789241504843_eng.pdf;jsessionid=876E08C843C919EA6CE890EB370 BEAB8? sequence $=1$. Accessed l February 2018.

Open Access This chapter is licensed under the terms of the Creative Commons Attribution 4.0 International License (http://creativecommons.org/licenses/ by $/ 4.0 /)$, which permits use, sharing, adaptation, distribution and reproduction in any medium or format, as long as you give appropriate credit to the original author(s) and the source, provide a link to the Creative Commons license and indicate if changes were made.

The images or other third party material in this chapter are included in the chapter's Creative Commons license, unless indicated otherwise in a credit line to the material. If material is not included in the chapter's Creative Commons license and your intended use is not permitted by statutory regulation or exceeds the permitted use, you will need to obtain permission directly from the copyright holder.

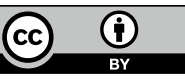

